\title{
Toward the scaling limit of the charm spectrum using domain wall and relativistic heavy quark
}

\author{
Hao Peng* \\ Department of Physics, Columbia University \\ E-mail: hp2151@columbia.edu
}

\begin{abstract}
We have extended the earlier relativistic heavy quark (RHQ) charm calculations performed on the $24^{3} \times 64,1 / a=1.73 \mathrm{GeV}, \mathrm{RBC} / \mathrm{UKQCD}$ lattice ensembles to the recently generated $32^{3} \times 64$, $1 / a=2.32 \mathrm{GeV}$ configurations. By calculating masses of charmonium and charm-strange states on these finer lattices, and comparing them to known experimental values, the RHQ parameters are determined and meson masses of interest are predicted. We have also examined the continuum limit of the resulting masses and present a new determination of the lattice spacing for these $32^{3} \times 64$ ensembles.
\end{abstract}

The XXVII International Symposium on Lattice Field Theory - LAT2009

July 26-31 2009

Peking University, Beijing, China

\footnotetext{
* Speaker.
} 


\section{Introduction}

The study of mesons containing heavy quarks plays an important role in particle physics by helping constrain the standard model parameters. The first-principle methods of lattice quantum chromodynamics (QCD) have been used to obtain some of the most accurate theoretical information so far, and connect to the experimental observations of those hadronic properties. However, conventional lattice calculations with heavy quarks meet special difficulties, since the relatively large masses of charm and bottom quarks $\left(m_{c}=1.25 \mathrm{GeV}, m_{b}=4.20 \mathrm{GeV}\right)$ make it impractical to work with lattice spacing sufficiently small to properly control discretization errors of $O(m a)$ [1]. To overcome this problem, a number of improved heavy quark actions are designed, including the heavy quark effective theory (HQET) and non-relativistic QCD (NRQCD), both of which have significant limitations. The HQET cannot be applied to quarkonia, while NRQCD has no continuum limit.

Another approach, which we have adopted in our heavy quark calculations, is the Fermilab or the relativistic heavy quark (RHQ) method [2, 3, 4], where axis-interchange asymmetric terms are added to usual lattice action, which takes the following form:

$$
S_{l a t}=\sum \bar{\psi}\left(m_{0} a+\gamma_{0} D_{0}+\zeta \vec{\gamma} \cdot \vec{D}-\frac{1}{2} r_{t}\left(D^{0}\right)^{2}-\frac{1}{2} r_{s}(\vec{D})^{2}+\sum_{\mu, v} \frac{i}{4} c_{p} \sigma_{\mu v} F_{\mu v}\right) \psi
$$

where

$$
\begin{gathered}
D_{\mu} \psi(x)=\frac{1}{2}\left[U_{\mu}(x) \psi(x+\hat{\mu})-U_{\mu}^{+}(x-\hat{\mu}) \psi(x-\hat{\mu})\right] \\
D_{\mu}^{2} \psi(x)=\left[U_{\mu}(x) \psi(x+\hat{\mu})+U_{\mu}^{+}(x-\hat{\mu}) \psi(x-\hat{\mu})-2 \psi(x)\right] \\
F_{\mu \nu} \psi(x)=\frac{1}{8} \sum_{s, s^{\prime}= \pm 1}\left[U_{s \mu}(x) U_{s^{\prime} v}(x+s \hat{\mu}) \times U_{-s \mu}\left(x+s \hat{\mu}+s^{\prime} \hat{v}\right) \times U_{-s^{\prime} v}\left(x+s^{\prime} \hat{v}\right)-H . c .\right] \psi(x) .
\end{gathered}
$$

This action has several advantages over the others. It works for all lattice spacing and approaches the standard relativistic action in the continuum limit as $m a$ becomes small. It supports nonperturbative methods. And, most important, it contains only 3 parameters to tune: the quark mass $m_{0} a$, an asymmetry parameter $\zeta$ describing the ratio of the coefficients of the spatial and temporal derivative, and $c_{p}$, a generalization of the Sheikholeslami and Wohlert term to the case of nonzero mass. By adjusting these coefficients properly, this action can accurately describe heavy quark systems. The hardronic masses computed in the resulting theory will contain errors no larger than $(\vec{p} a)^{2}[2,3,4]$.

The first thing that needs to be done with the RHQ action is to determine the 3 RHQ parameters. We do this here by matching to the experimental data, working in a small parameter range where the dependence on $m_{0} a, \zeta, c_{p}$ is approximately linear. Then we can use the 3 coefficients to predict meson masses in which we are interested. Both of the above require us to know the lattice spacing from the beginning. However, if the lattice spacing is unknown, we can also work it out from the RHQ action, by just determining it as a 4th RHQ parameter. We can then check whether it is consistent with results from other approaches. Actually the whole work described here has already been successfully done on the RBC/UKQCD $24^{3} \times 64$ lattice ensembles [5]. The RHQ parameters and lattice spacing there are calculated and extrapolated to the physical light quark mass limit, with $m_{0} a=0.251(9), c_{p}=2.091(17), \zeta=1.242(10), a_{24}^{-1}=1.730(23) \mathrm{GeV}$. 
This calculation also accurately predicted the masses of the $\chi_{c 0}$ and $\chi_{c 1}$ mesons, which are $m_{\chi_{c 0}}=$ $3.424(11) \mathrm{GeV}(\exp .=3.415 \mathrm{GeV})$ and $m_{\chi_{c 1}}=3.502(14) \mathrm{GeV}(\exp .=3.511 \mathrm{GeV})$ respectively [5], within 1 standard deviation of the experimental results.

\section{Methods}

We have carried out a series of calculations of heavy-heavy and heavy-strange correlators in charm system using the RHQ action of eq. 1.1, on a 1k-node partition of the QCDOC machines in the RIKEN BNL Research Center (RBRC). Specifically, we calculate the pseudoscalar (PS), vector $(\mathrm{V})$, scalar (S), and axial-vector (AV) meson masses, which are fitted from the time dependence of the heavy-heavy correlators, and the pseudoscalar, vector masses from heavy-strange systems. Following our old convention in Ref. [6], we work with the following mass combinations, spinaverage, $m_{s a}^{h h}=\frac{1}{4}\left(m_{p s}^{h h}+3 m_{v}^{h h}\right), m_{s a}^{h s}=\frac{1}{4}\left(m_{p s}^{h s}+3 m_{v}^{h s}\right)$, hyperfine splitting, $m_{h s}^{h h}=m_{v}^{h h}-m_{p s}^{h h}, m_{h s}^{h s}=$ $m_{v}^{h s}-m_{p s}^{h s}$, spin-orbit average and splitting, $m_{s o a}^{h h}=\frac{1}{4}\left(m_{s}^{h h}+3 m_{a v}^{h h}\right), m_{s o s}^{h h}=m_{a v}^{h h}-m_{s}^{h h}$, and the mass ratio, $m_{1} / m_{2}$, from the dispersion relation, $E^{2}=m_{1}^{2}+\frac{m_{1}}{m_{2}} p^{2}$, with $m_{1}$ the rest mass and $m_{2}$ the kinetic mass. By matching $m_{s a}^{h h}, m_{h s}^{h h}$ to the experiment results and requiring $m_{1} / m_{2}=1$, we can then determine the three parameters $m_{0} a, \zeta$ and $c_{p}$.

To start the matching procedure, we choose a sufficiently small region in the neighborhood of the physical point in our parameter space, so that the resulting meson masses are well fit by a simple linear dependence on the heavy quark parameters:

$$
Y=A+J \cdot X
$$

where $\mathrm{X}$ is a vector of input RHQ parameters, $X^{T}=\left(m_{0} a, c_{p}, \zeta\right)$, and $\mathrm{Y}$ is made up of computed masses or mass ratios, $Y^{T}=\left(m_{s a}^{h h} a, m_{h s}^{h h} a, m_{s a}^{h s} a, m_{h s}^{h s}, m_{s o a}^{h h}, m_{s o s}^{h h}, m_{1} / m_{2}\right)$. The quantities A and J represent constant and linear terms in our linear approximation, and can be determined by minimizing $\chi^{2}$ defined as:

$$
\chi^{2}=\sum(A+J \cdot X-Y)^{T} \cdot W^{-1} \cdot(J \cdot X+A-Y)
$$

where $\mathrm{W}$ is the jackknifed error matrix:

$$
W_{d, d^{\prime}}=\sum<\left(Y_{d}-\bar{Y}_{d}\right)\left(Y_{d^{\prime}}-\bar{Y}_{d^{\prime}}\right)>
$$

and the resulting RHQ parameters are determined by solving a set of linear equations and can be written explicitly as

$$
X_{c}=\left(J^{T} \cdot W^{-1} \cdot J\right)^{-1} \cdot J^{T} \cdot W^{-1} \cdot\left(Y_{\exp }-A\right) .
$$

The error on $X_{c}$ is calculated from the jackknifed results of $J$ and $A$ which represent the statistical fluctuations in our lattice ensembles.

In our actual calculation, we pick up 7 sets of input RHQ parameters shown in Figure 1. Then the quantities $A$ and $J$ are directly determined using the following expressions:

$$
\begin{gathered}
J_{d, i}=\frac{1}{2 \Delta_{i}}\left(Y_{d}\left(\bar{X}+\Delta_{i}\right)-Y_{d}\left(\bar{X}-\Delta_{i}\right)\right) \\
A_{d}=\frac{1}{6} \sum Y_{d}-\sum J_{d, i} \bar{X}_{i} .
\end{gathered}
$$




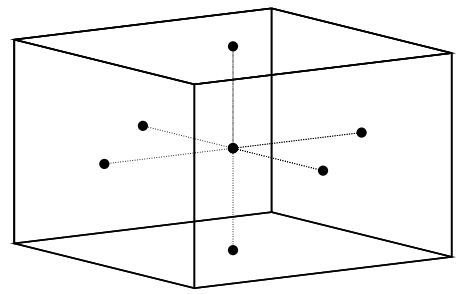

Figure 1: The distribution in the 3-parameter space $\left(m_{0} a, c_{p}, \zeta\right)$ of the 7 -set Cartesian data. Throughout our calculation, the central point is fixed at $\bar{X}=$ $(-0.06,1.5327,1.1774)$, and the corresponding increments are $\Delta=(0.1,0.1,0.02)$ respectively.

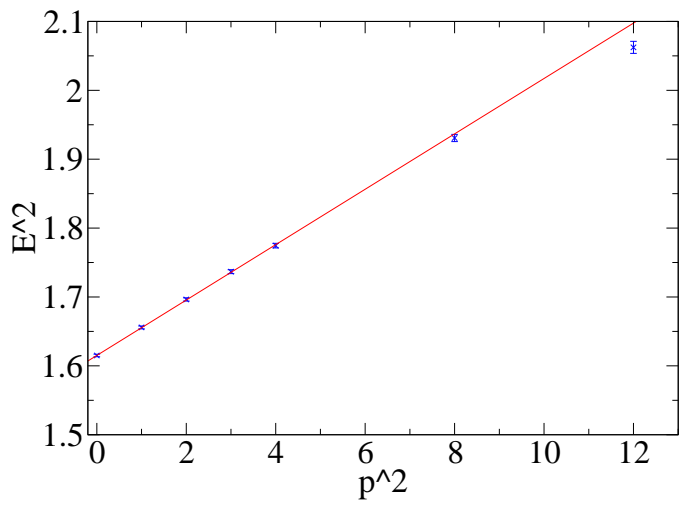

Figure 2: Momentum dependence of $E_{p s}$ which is used to evaluate the mass ratio $m_{1} / m_{2}$.

To determine the 3 unknown RHQ parameters, we need only $3 Y_{d}^{\prime} s$. In fact, we select the meson masses that are most precisely extracted from the heavy correlators, $Y^{T}=\left(m_{s a}^{h h} a, m_{h s}^{h h} a, m_{1} / m_{2}\right)$, and match to the experimental results, $Y_{\text {exp }}^{T}=\left(\frac{1}{4}\left(m_{\eta_{c}}+3 m_{j / \psi}\right) a,\left(m_{j / \psi}-m_{\eta_{c}}\right) a, m_{1} / m_{2}\right)$, to calculate $\left(m_{0} a, c_{p}, \zeta\right)$. The remaining components in $Y$ are used to make predictions in the following way:

$$
Y_{\text {pred }}=A+J \cdot X_{c} .
$$

To evaluate the lattice spacing for the $32^{3} \times 64$ ensembles, we treat $a$ as the 4 th input parameter, set $Y^{T}=\left(m_{s a}^{h h} a, m_{h s}^{h h} a, m_{s a}^{h s} a, m_{1} / m_{2}\right)$, and follow the same steps in the 3-parameter case.

\section{Results and Discussions}

The configurations used in our calculations are generated by the RBC-UKQCD collaboration, with properties shown in Table 1. We calculated all correlators on the QCDOC machines in the RBRC. We then look at the effective mass plots as shown in Figure 3, find a reasonable plateau and extract the meson masses by doing a hyperbolic cosine fit over that region. The ratio of the rest mass to the kinetic mass is evaluated from the dispersion relation of the pseudo-scalar meson $\left(\eta_{c}\right)$, which has a longer plateau than other meson, and is shown is Figure 2. From this plot we can see an obvious linear dependence for the 5 smallest momenta, $p^{2}=\left(\frac{2 \pi}{L}\right)^{2}(0,1,2,3,4)$, which allows us to apply linear regression to obtain the ratio. Except when evaluating the lattice spacing in the last section, we use the value $a^{-1}=2.32 \mathrm{GeV}$, which can be found in Ref. [7].

\begin{tabular}{ccccc}
\hline \hline volume & $L_{s}$ & $\left(m_{\text {sea }}, m_{s}\right)$ & Trajectory(step) & \# of configs \\
\hline $32^{3} \times 64$ & 16 & $(0.004,0.03)$ & $500-3420(20)$ & 147 \\
$32^{3} \times 64$ & 16 & $(0.006,0.03)$ & $500-3820(20)$ & 167 \\
$32^{3} \times 64$ & 16 & $(0.008,0.03)$ & $500-2960(20)$ & 124 \\
\hline \hline
\end{tabular}

Table 1: The dynamical $2+1$ flavor $\beta=2.25$ lattice configurations used for our charmonium and charmstrange calculations. The time interval between 2 measurements is 40 time units. For each case a box source was located at the origin in the lattice. The box source size is chosen to be 6 , giving almost the same physical volume as that used for the $24^{3}$ configuration. 

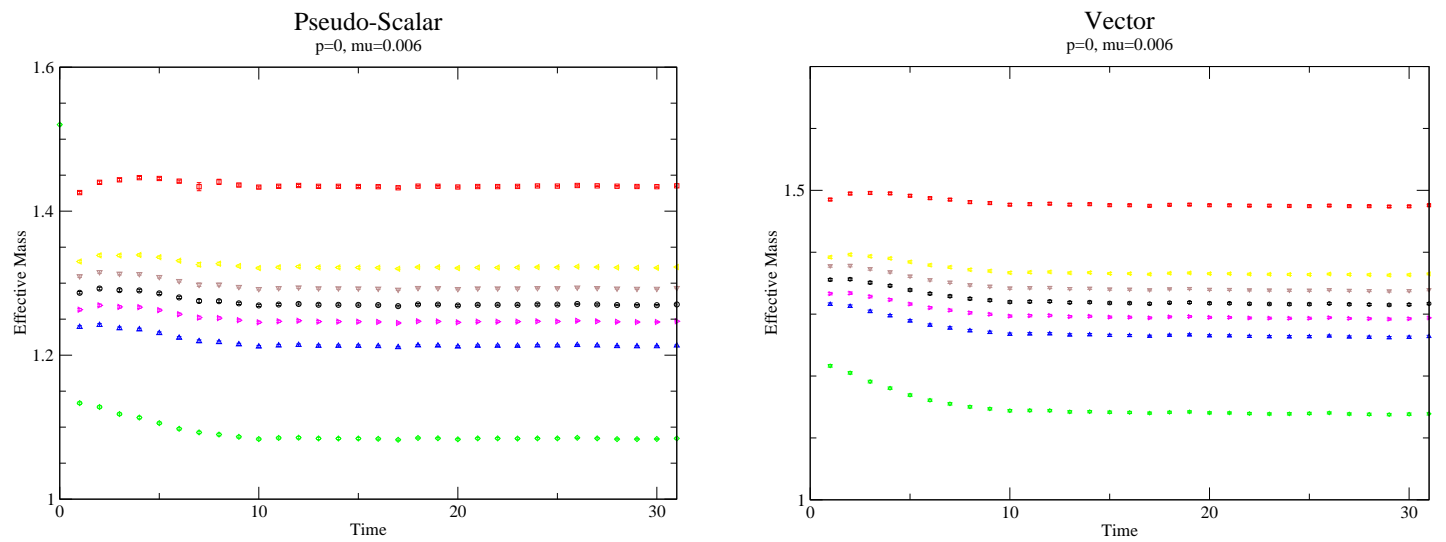

Figure 3: Sample effective mass plots of pseudo-scalar and vector states from charmonium correlators. The fitting ranges of the effective masses are chosen as 10-30 (ps), 13-30 (v) respectively.

\subsection{RHQ parameters and predictions}

The resulting RHQ parameters and corresponding meson masses predicted from them are listed in Table 2. Here we ignore the results for the scalar $\left(\chi_{c 0}\right)$ and axial-vector $\left(\chi_{c 1}\right)$ states due to lack of statistics, although some preliminary results were presented at the lattice conference.

\begin{tabular}{c|ccc|cc}
\hline \hline$m_{\text {sea }}$ & $m_{0} a$ & $C_{p}$ & $\zeta$ & $m_{D_{s}}(\mathrm{GeV})$ & $m_{D_{s}^{*}}(\mathrm{GeV})$ \\
\hline 0.004 & $0.050(15)$ & $1.787(38)$ & $1.137(10)$ & $1.976(1)$ & $2.106(1)$ \\
0.006 & $0.021(14)$ & $1.705(37)$ & $1.153(10)$ & $1.972(1)$ & $2.102(1)$ \\
0.008 & $0.021(14)$ & $1.674(37)$ & $1.147(11)$ & $1.979(2)$ & $2.099(1)$ \\
\hline \hline
\end{tabular}

Table 2: RHQ parameters for the $32^{3} \times 64$ ensembles and meson mass predictions for different $m_{\text {sea }}$.

We then apply a linear extrapolation on these quantities to the physical light quark mass limit, and obtain the final RHQ parameters which will be used in future $B-\bar{B}, D-\bar{D}$ mixing calculations, etc, as well as meson masses that can be used to check whether our methods are successful. The extrapolation results are shown in Figure 4 and 5.

\subsection{Continuum limit}

We compare our predicted masses of $D_{s}$ and $D_{s}^{*}$ for the $24^{3}$ and $32^{3}$ ensembles to the experimental values shown in Table 3. Our results agree with the experimental values within an error of $6 \mathrm{MeV}$, which means our RHQ methods with 3 input parameters work quite successfully.

\begin{tabular}{c|ccc}
\hline \hline meson & $32^{3} \mathrm{RHQ}(\mathrm{GeV})$ & $24^{3} \mathrm{RHQ}(\mathrm{GeV})$ & Exp.(GeV) \\
\hline$m_{D_{s}}$ & $1.9742(24)$ & $1.964(3)$ & $1.9682(5)$ \\
$m_{D_{s}^{*}}$ & $2.1123(19)$ & $2.113(7)$ & $2.1120(6)$ \\
\hline
\end{tabular}

Table 3: Predicted masses of $D_{s}$ and $D_{s}^{*}$ for both the $32^{3}$ and $24^{3}$ ensembles as well as the experimental values. Note, the heavy-strange measurements on the $32^{3}$ ensembles were separated by only 4 units, so the errors are likely underestimated. An improved calculation is now under way.

We can now use the $24^{3}$ and $32^{3}$ results, together with experimental quantities, to discuss the scaling limit issue. Since we know that the RHQ lattice calculation removes all discretization errors 

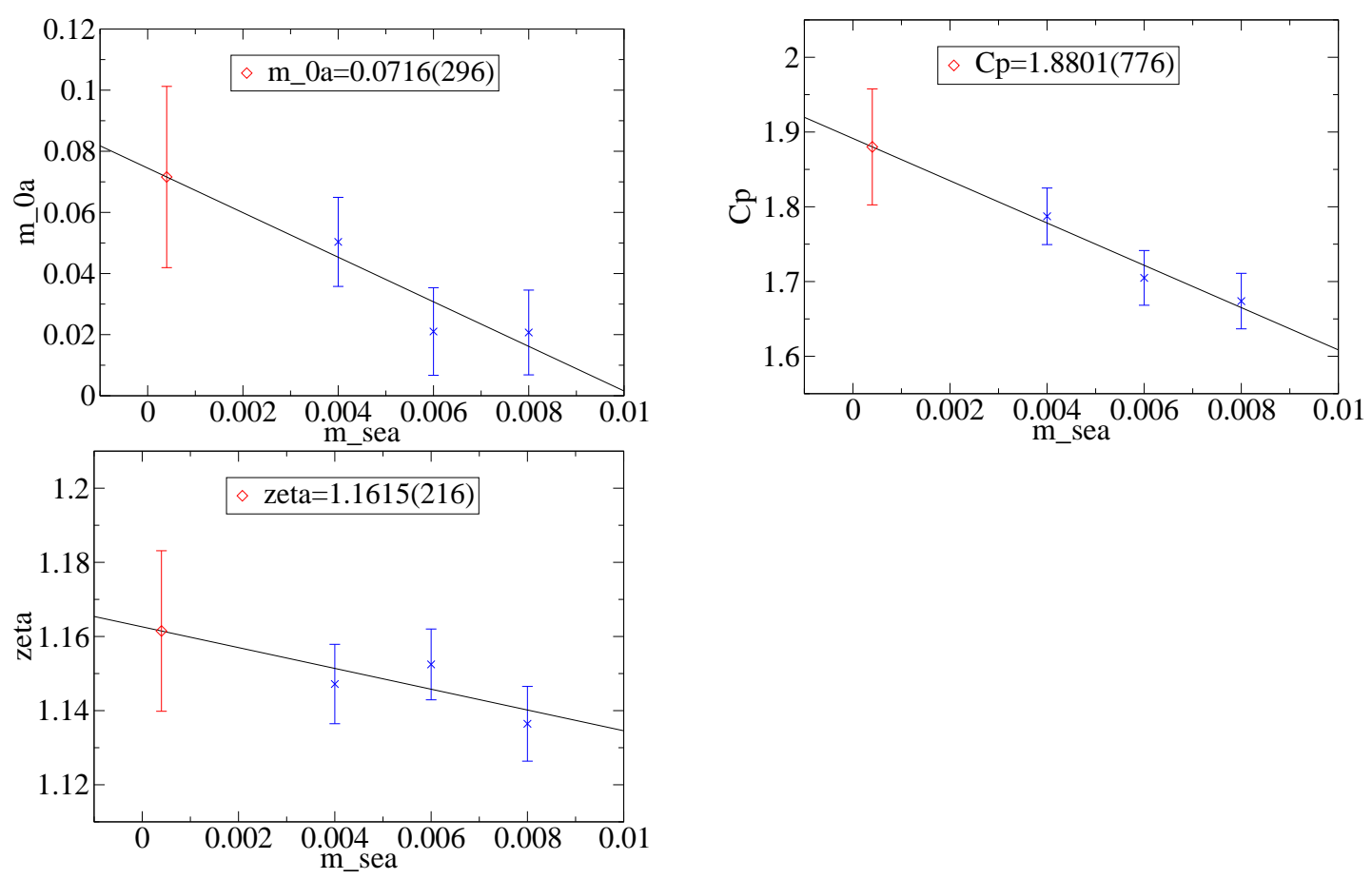

Figure 4: Extrapolation of the RHQ parameters to the physical light quark mass limit at $m_{\text {sea }}=0.000399$.
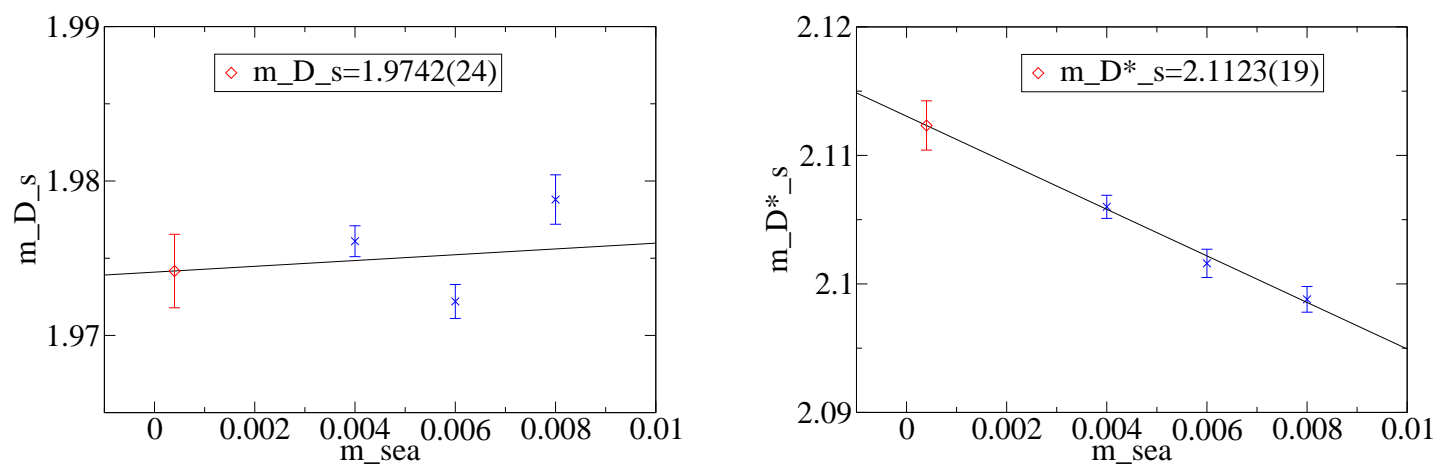

Figure 5: Extrapolation of the heavy-strange meson masses to the physical light quark mass limit.

of $O(a)$, so that $O\left(a^{2}\right)$ errors are expected in the result. However, from Figure 6, the 3 points for both $D_{s}$ and $D_{s}^{*}$ are not exactly on a straight line, instead higher order deviations, probably $O\left(a^{3}\right)$ needs considering according to our preliminary RHQ calculations. This is expected because the coefficient of the $O\left(a^{2}\right)$ term will be a function of $m_{c} a$.

\subsection{Lattice spacing Determination}

The lattice spacings calculated from all 3 ensembles are shown in Table 4. We do the same linear extrapolation, shown in Figure 7, and obtain the final result at the physical light quark mass limit, $a^{-1}=2.296(39) \mathrm{GeV}$, which is consistent with the $2.32 \mathrm{GeV}$ result from Ref. [7]. 


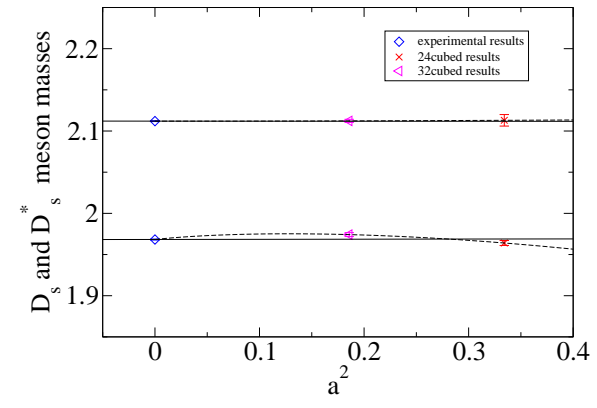

Figure 6: Strange-charm meson masses measured as a function of $a^{2}$. The upper is $D_{s}^{*}$ and the lower is $D_{s}$. The solid line is fit to $a^{2}$, while the dashed curve is fit to $a^{3}$

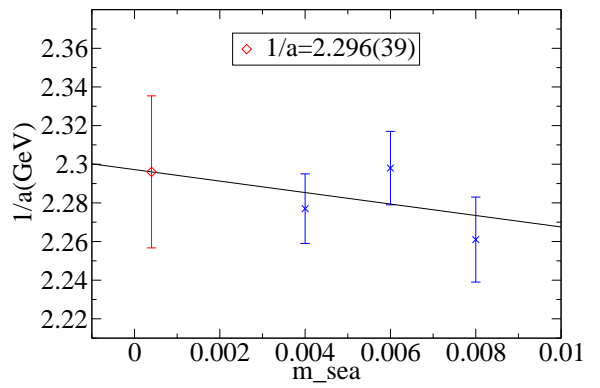

Figure 7: Extrapolation of the lattice spacing to the physical light quark mass limit

\begin{tabular}{c|cccc}
\hline \hline$m_{\text {sea }}$ & 0.004 & 0.006 & 0.008 & 0.000399 \\
\hline $1 / a$ & $2.277(18)$ & $2.298(19)$ & $2.261(22)$ & $2.296(39)$ \\
\hline \hline
\end{tabular}

Table 4: The lattice spacing evaluated from ensembles with $m_{\text {sea }}=0.004,0.006,0.008$, and the result in the physical light quark mass limit.

\section{Acknowledgements}

Our work was supported by DOE Grant No.DE-FG02-92ER40699. I would like to thank Norman Christ, Robert Mawhinney, Chulwoo Jung, Michael Endres, Matthew Lightman, Xiaoyong Jin, Qi Liu, Shinji Takeda, Taku Izubuchi and all members in the RBC collaboration for their help throughout the course of the work. I would particularly appreciate Min Li for his help on starting the RHQ calculations. I also thank the RBRC for providing facilities essential to this work.

\section{References}

[1] A. S. Kronfeld, Heavy quarks and lattice QCD, Nucl. Phys. Proc. Suppl. 129 (2004) 46-59, [hep-lat/0310063].

[2] A. X. El-Khadra, A. S. Kronfeld, and P. B. Mackenzie, Massive Fermions in Lattice Gauge Theory, Phys. Rev. D55 (1997) 3933-3957, [hep-lat/9604004].

[3] S. Aoki, Y. Kuramashi, and S.-i. Tominaga, Relativistic heavy quarks on the lattice, Prog. Theor. Phys. 109 (2003) 383-413, [hep-lat/0107009].

[4] N. H. Christ, M. Li, and H.-W. Lin, Relativistic heavy quark effective action, Phys. Rev. D76 (2007) 074505, [hep-lat/0608006].

[5] M. Li and H.-W. Lin, Charm spectroscopy on dynamical 2+1 flavor domain wall fermion lattices with a relativistic heavy quark action, PoS LAT2007 (2007) 117, [0 710 . 0910].

[6] H.-W. Lin and N. Christ, Non-perturbatively determined relativistic heavy quark action, Phys. Rev. D76 (2007) 074506, [hep-lat/0608005].

[7] RBC and UKQCD Collaboration, R. Mawhinney, Nlo and nnlo chiral fits or 2+1 flavor dwf ensembles, PoS LAT2009 (2009) 081. 